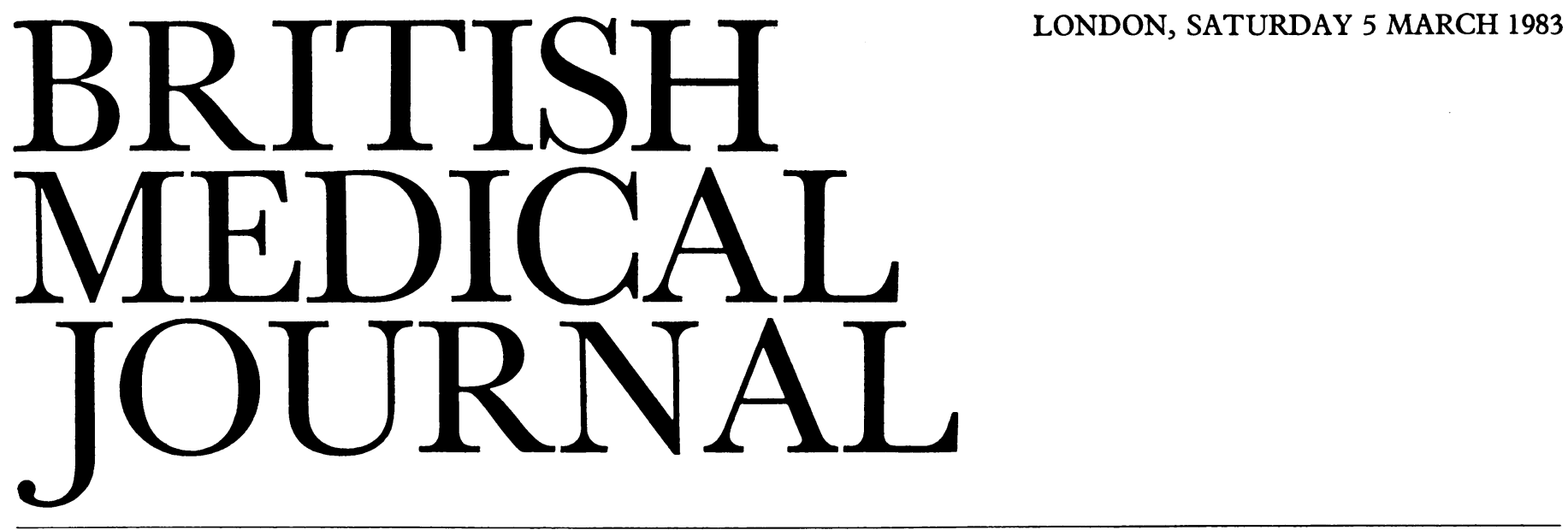

\title{
Interferon and cancer
}

The anticancer potential of interferon has generated tremendous interest over the past few years. Originally discovered in 1957 as a protein which interfered with viral infection, interferon has been the focus of much recent research in both the laboratory and clinic. Early work was hampered by the small quantities of naturally occurring interferon available for study, but the full power of modern molecular biology has now been applied to the problem of producing adequate amounts for clinical trial. The genes coding for human interferon have been cloned and recombined with bacterial plasmids. ${ }^{1}$ Such plasmids have been used to infect bacteria which could then be grown in culture to obtain highly purified interferon, and two pharmaceutical houses are now producing large amounts of "recombinant" interferon.

We have learnt much about the biology of the interferon system. After reaching its target cell interferon binds to a surface receptor and sets in motion a series of secondary messengers culminating in a change in the cell's general metabolic state. $^{2}$ These changes inhibit the ability of viruses to replicate using the genetic machinery of the host cell and also have a complex effect on cell growth and division. Interferon also has profound effects on the immune system, acting under some circumstances as a lymphocyte hormone. ${ }^{3}$

A further problem in understanding interferons and in assessing their efficacy is their heterogeneity, for there are no fewer than three families of interferon: $\alpha$-predominantly produced by leucocytes, $\beta$-by fibroblasts, and $\gamma$-by antigen or mitogen stimulated lymphocytes. Gene cloning has shown that in addition there are subtypes of the $\alpha$ interferon resulting in a total of at least 14 different molecular species making up the interferons. ${ }^{1}$ While one of their physiological functions is defence against viral infection, the other functions of these disparate molecules are not clear. We do not know, for example, whether inhibition of cellular replication is a feature of all or only a subgroup of these molecules.

But what of the clinical uses? The early preparations of relatively impure interferon were shown to have some effect in causing objective regression of tumours in patients with myeloma, breast cancer, and non-Hodgkin's lymphoma. ${ }^{4}$ These responses tended, however, to be partial and transient. The pioneers in this work hoped that they might get better results if they gave patients greater quantities of interferon. Unfortunately, the early preparations had serious side effects, principally fever, malaise, and fatigue. Because of the impurity of the interferons used at that time it was uncertain whether these effects were intrinsic to interferon or were a result of contaminants. Answers to these questions are beginning to emerge now that very pure recombinant interferon is available for large scale clinical trials. Firstly, the recombinant interferons also cause objective regression in certain types of tumour and appear to be at least as biologically active as the naturally occurring preparation. Clearly, however, pure interferon has profound side effects which limit the dose that can be given. Careful pharmacokinetic studies have shown that patients cannot tolerate doses beyond 36 million units a day for longer than one month. Side effects at this dosage include anorexia, loss of weight, and central nervous system toxicity, which may present with features of a metabolic encephalopathy. Changes in the electroencephalogram observed in this syndrome include excess slow wave activity. ${ }^{56}$ All this makes the administration of interferon an arduous task for the clinical investigator.

Many trials are now in progress with recombinant interferon. So far regression of tumours has been observed consistently only in patients with myeloma, non-Hodgkin's lymphoma, and breast cancer. A few patients have had well documented objective responses in diseases such as melanoma, renal cell carcinoma, lung cancer, and Kaposi's sarcoma (S Krown, personal communication). The response rate in some of these tumours is low-less than $20 \%$-but the patients studied in these early phase II trials have all had advanced disease, resistant to conventional treatment, so that any response is encouraging. Most of these responses have been partial, a reduction in tumour size rather than complete disappearance, and there are many problems in assessing tumour load in an individual patient. Nevertheless, most of these responses are probably real and interferon is having an inhibitory effect on the growth of at least some tumours, either directly or through the immune system.

The outlook for patients with the common solid tumours appears to have reached a plateau despite intense effort with conventional cytotoxic chemotherapy. Against that background further investigation of the anticancer potential of interferon seems reasonable. If indeed interferon works through an immunomodulatory mechanism then it might benefit patients with earlier disease in an adjuvant setting. Despite the low response rate when used as a single agent in advanced disease the combination of interferon with other drugs might be useful. Investigators will probably be prudent and work only with the highly purified preparations produced by genetic engineering techniques-so removing the possibility that impurities may be causing any antitumour effects as well as being able to reproduce precisely the production methods. Problems with varia- 
tion in the activity of earlier preparations from one batch to another have caused confusion in comparing results.

Clearly interferon is not to cancer what penicillin was to bacterial infection. Nevertheless, it may still have a part to play in the treatment of cancer and only careful clinical and laboratory research in centres familiar with the rigours of such investigation can determine its eventual role in clinical oncology.

KAROL SIKORA

Honorary consultant in radiotherapy and oncology

HOWARD SMEDLEY

Senior clinical scientist and honorary senior registrar in radiotherapy and oncology

Ludwig Institute for Cancer Research,

Addenbrooke's Hospital,

Cambridge CB2 2QH

${ }^{1}$ Sehgal PB. The interferon genes. Biochim Biophys Acta 1982;695:17-34.

${ }^{2}$ Williams BRG, Golgher RR, Hovanessian AG, Kerr IM. The involvement of the 2-5A (ppp'p5'A2'p5'A) system and protein kinases in the antiviral and anticellular effects of interferon. In: Collier LH, Oxford J, eds. Development in antiviral therapy. London: Academic Press, 1980: 173-87.

${ }^{3}$ Saksela E. Interferon and natural killer cells. In: Gresser I, ed. Interferon. Vol 3. London: Academic Press, 1981:45.

4 Sikora K. Does interferon cure cancer? Br Med f 1980;281:855-8.

${ }^{5}$ Smedley H, Katrak M, Sikora K, Wheeler T. Neurological effects of recombinant human interferon. Br Med $\mathcal{F} 1983 ; 286: 262-4$.

6 Rohatiner AZ, Prior PF, Burton AL, Smith AT, Balkwill FR, Lister TA. Central nervous system toxicity of interferon. Br $\mathcal{f}$ Cancer (in press).

\section{Thyroid surgery for Graves' disease}

In most patients with Graves' disease the hyperthyroidism is characterised by alternating episodes of relapse and remission over several years. A euthyroid state may readily be restored by treatment with drugs such as carbimazole; but antithyroid drugs have little or no influence on the natural history of the disorder despite their immunosuppressive action in reducing the serum concentration of antibodies against thyroid stimulating hormone (TSH) receptors. ${ }^{1}$ Indeed, at least two thirds of all patients treated with antithyroid drugs for six to 18 months will relapse, usually within one to two years of stopping treatment. $^{2}$ The other third remain in prolonged remission and may even develop hypothyroidism in future years, ${ }^{3}$ a sequence of events first recognised last century. ${ }^{4}$

Clearly a single course of antithyroid drugs would be ideally suited to the "remission" group but would be inappropriate treatment for the "relapse" group. Unfortunately the course of hyperthyroidism cannot be predicted in individual patients when they present, though in some populations those who are HLA-DR3 positive and have serum antibodies against TSH receptors after a six month course of carbimazole are very likely to relapse in the short term. ${ }^{5}$ These observations require confirmation, but in the mean time neither HLA typing nor the measurement of antibodies against TSH receptors is widely available. With few exceptions, thereforesuch as in pregnancy, childhood, and patients who depend on their vocal cords for a living-many British authorities prefer to advise subtotal thyroidectomy as the initial treatment for Graves' disease in patients aged under 40 . Whether surgery is the only alternative to treatment with antithyroid drugs is another matter; in the United States radioactive iodine is used much more liberally in patients of reproductive age.
What is the best preparation for thyroid surgery? The long established practice has been with carbimazole and potassium iodide. In recent years various studies have shown that propranolol alone is an effective alternative. ${ }^{6-8}$ The consensus is, however, that the greater flexibility of operation date and reduced operative blood loss given by this method are more than offset by the risk of surgery in patients with raised thyroid hormone concentrations and in a catabolic state. If, for business or other reasons, surgery cannot be delayed for the six to eight weeks necessary for conventional preparation, potassium iodide should be added to propranolol or another non-selective beta-blocker for 10 days before subtotal thyroidectomy. This treatment usually lowers the concentrations of hormones to the normal range. ${ }^{910}$

In experienced hands the results of subtotal thyroidectomy are better than those achieved with antithyroid drugs. At the end of the first year some $80 \%$ of patients are euthyroid, $15 \%$ are permanently hypothyroid, and 5\% have relapsed. ${ }^{8}$ The incidence of postoperative hypothyroidism has been overestimated in the past because it was not widely appreciated that the thyroid failure might be temporary. Low serum concentrations of total thyroxine and raised concentrations of TSH have been recorded in about a third of patients three months after surgery with or without associated symptoms and signs of mild hypothyroidism-but by the sixth month thyroid hormone concentrations have usually returned to normal spontaneously. ${ }^{8}$ Permanent hypothyroidism must not, therefore, be diagnosed before six months after operation; and if for clinical reasons thyroxine has to be prescribed earlier an attempt should be made to discontinue it later. Thyroid failure after the first year is well documented, as is relapse of hyperthyroidism even as long as 30 years or more after an apparently successful operation. ${ }^{11}$

Clearly patients with Graves' disease treated surgically need long term review, but how often should such patients be followed up? Can the intervals at which they are seen be determined on the basis of tests of thyroid function ? Such a policy has proved possible, for example, in thyrotoxic patients successfully treated with iodine-131. After measuring serum concentrations of TSH two groups emerged, one with raised concentrations who developed hypothyroidism at a rate of $5 \%$ each year and required yearly review, and a second with normal concentrations who were at much less risk of thyroid failure and could be reviewed every three to five years. ${ }^{12}$ After subtotal thyroidectomy raised concentrations of TSH are common in euthyroid patients. ${ }^{13}{ }^{14}$ In a recent study ${ }^{15}$ TSH concentrations were raised in $70 \%$ of euthyroid patients one year after operation, but none developed hypothyroidism in the ensuing four years, and in some concentrations of $\mathrm{TSH}$ returned to normal. Furthermore, a raised TSH concentration did not preclude the subsequent development of recurrent hyperthyroidism. Unfortunately, therefore, TSH cannot be used to predict thyroid function after surgery for Graves' disease, and yearly review either by the hospital clinic or by the general practitioner (prompted by computer ${ }^{16}$ ) must continue for all patients. Nevertheless, it is not necessarily an ill wind. The hard pressed clinical biochemist can rejoice, as in this particular group of patients measurement of $\mathrm{TSH}$ is indicated only to confirm the validity of a low serum concentration of thyroxine after the third postoperative month and before starting permanent replacement treatment.

Senior Lecturer in Medicine,

ANTHONY D TOFT

Royal Infirmary

Edinburgh EH3 9YW 